\title{
Development of a Web-Based Nonoperative Small Bowel Obstruction Treatment Pathway App
}

\author{
Heather Lyu ${ }^{1,2}$ Caitlin Manca ${ }^{2}$ Casey McGrath ${ }^{2}$ Jennifer Beloff ${ }^{2}$ Nina Plaks $^{3}$ Anatoly Postilnik ${ }^{3}$
} Amanda Borchers ${ }^{3}$ Nicasio Diaz ${ }^{3}$ Sean McGovern ${ }^{3}$ Joaquim Havens ${ }^{1,2}$ Allen Kachalia ${ }^{4}$

Adam Landman ${ }^{1,3,5}$

\footnotetext{
1 Department of Surgery, Brigham and Women's Hospital, Boston, Massachusetts, United States

2 Department of Quality and Safety, Brigham and Women's Hospital, Boston, Massachusetts, United States

3 Partners HealthCare, Somerville, Massachusetts, United States

${ }^{4}$ Department of Biomedical Informatics, Harvard Medical School, Boston, Massachusetts, United States

${ }^{5}$ Armstrong Institute for Patient Safety and Quality, Johns Hopkins Hospital, Baltimore, Maryland, United States
}

Appl Clin Inform 2020;11:535-543.

\begin{abstract}
Keywords

- pathways

- digital health

- Web-based application

- EHR

- surgery

Objective An electronic pathway for the management of adhesive small bowel obstruction (SBO) was built and implemented on top of the electronic health record. The aims of this study are to describe the development of the electronic pathway and to report early outcomes.

Methods The electronic SBO pathway was designed and implemented at a single institution. All patients admitted to a surgical service with a diagnosis of adhesive SBO were enrolled. Outcomes were compared across three time periods: (1) patients not placed on either pathway from September 2013 through December 2014, (2) patients enrolled in the paper pathway from January 2017 through January 2018, and (3) patients enrolled in the electronic pathway from March through October 2018. The electronic SBO pathway pulls real-time data from the electronic health record to prepopulate the evidence-based algorithm. Outcomes measured included length of stay (LOS), time to surgery, readmission, surgery, and need for bowel resection. Comparative analyses were completed with Pearson's chi-squared, analysis of variance, and Kruskal-Wallis tests.

Results There were 46 patients enrolled in the electronic pathway compared with 93 patients on the paper pathway, and 101 nonpathway patients. Median LOS was lower in both pathway cohorts compared with those not on either pathway (3 days [range 1-11] vs. 3 days [range 1-27] vs. 4 days [range 1-13], $p=0.04$ ). Rates of readmission, surgery, time to surgery, and bowel resection were not significantly different across the three groups.

Conclusion It is feasible to implement and utilize an electronic, evidence-based clinical pathway for adhesive SBOs. Use of the electronic and paper pathways was associated with decreased hospital LOS for patients with adhesive SBOs.
\end{abstract}

Address for correspondence Heather Lyu, MD, MBI, 75 Francis Street, Boston, MA 02115, United States (e-mail: hlyu@bwh.harvard.edu). received

March 30, 2020

accepted after revision

July 1,2020 (c) 2020 Georg Thieme Verlag KG Stuttgart · New York
DOI https://doi.org/

10.1055/s-0040-1715478.

ISSN 1869-0327. 


\section{Background and Significance}

Time and task-oriented clinical pathways are being utilized widely across the United States with implementation of pathways in over $80 \%$ of hospitals nationwide. ${ }^{1,2}$ Implementation of clinical pathways has increased since a landmark article published in 1995 described their impact on efficiency and quality in high-volume settings. ${ }^{3}$ Since then, multiple studies have demonstrated the impact of clinical pathways on reduced inhospital complications, hospital length of stay (LOS), and costs of care without increasing patient mortality or morbidity. ${ }^{3,4}$ One study showed that a novel electronic Enhanced Recovery After Surgery pathway was successful in reducing costs by $1.28 \%$ or $\$ 2,730$ per surgical encounter or patient. ${ }^{5}$ Pathways are tailored to standardize care for specific problems or procedures. This is exemplified in a study demonstrating how structured electronic health record (EHR) data can be used to visualize the algorithms behind the diagnoses of abdominal pain. ${ }^{6}$ The development of pathways is complex and requires multidisciplinary participation to build a treatment plan with smooth criteria-based progression of clinical events.

At our institution, a Clinical Pathways Program was established to merge national guidelines with local initiatives to standardize patient care based on evidence at the institutional level. One of the first pathways developed and implemented was the small bowel obstruction (SBO) pathway which was designed to standardize care for patients admitted with a diagnosis of adhesive SBO. SBOs are common in patients after surgery with adhesive obstructions accounting for $4 \%$ of all emergency department admissions, $20 \%$ of emergency surgical procedures, and over 300,000 hospitalizations totaling $\$ 1.3$ billion dollars in cost to the U.S. health care system. ${ }^{7,8}$ The majority of SBOs resolve without surgery and the mainstay of treatment for this condition is bowel rest with nasogastric tube (NGT) decompression of the stomach. ${ }^{8}$ However, therapies for SBOs remain widely variable and a review of current practice patterns showed extensive inconsistencies in SBO treatment, particularly in the trial of nonoperative management. ${ }^{9}$ While practice guidelines recommend limiting nonoperative therapy to 3 to 5 days, surgeons often extend this duration leading to delayed definitive management and worse outcomes. ${ }^{10}$

The World Society of Emergency Surgery released a new treatment algorithm called the water-soluble contrast challenge (WSCC) which requires the administration of hyperosmolar, water-soluble contrast (Gastrografin) through the NGT. ${ }^{11,12}$ The WSCC has been proven to be safe and feasible. Gastrografin has been shown to have therapeutic value for SBOs with faster resolution of SBOs and decreased hospital LOS. $^{13,14}$ Our institution adopted the WSCC in 2015 and tailored the pathway to local availability by replacing Gastrografin with a low osmolarity water-soluble contrast called Omnipaque. Initial studies of the SBO pathway using Omnipaque have similarly demonstrated reductions in hospital LOS. ${ }^{15}$

Given the initial success of the paper pathway, we sought to create an electronic version. We reviewed our EHR vendor's functionality and did not think it adequately met our functional and workflow requirements. Mandl and Kohane describe a future app ecosystem where health care organizations will be able to add applications on top of their EHRs. ${ }^{16}$ We designed, developed, and implemented a custom Web application that integrates directly into our EHR, drawing patient data using application programming interfaces (APIs), and displaying the content within the EHR, while maintaining user and patient context.

The intended purposes of the electronic SBO pathway included real-time visualization to provide clinical-decision support and provision of a platform for administrators and researchers to prospectively collect process and compliance metrics. In this study, we describe the design and development of the electronic SBO pathway that integrates into the EHR and report early outcomes including LOS, readmission, surgery, time to surgery (TTS), and need for bowel resection.

\section{Methods}

Both the electronic and paper SBO pathways were designed and implemented for patients treated on the trauma and acute care surgery service at a single high-volume academic institution. To limit variability of care provided by various surgical providers and services, all patients admitted with adhesive SBOs on other surgical or medical services were excluded. A commercially available, enterprise-based electronic medical record system (Epic Systems, Verona, Wisconsin, United States) was implemented in this hospital in 2015.

\section{Development of the SBO Pathway Application}

The paper-based SBO pathway was used as the basis for the electronic version (-Fig. 1). The electronic SBO pathway application was custom-built by a team of software developers. An iterative design and development process was used by a multidisciplinary team of clinicians, quality and safety experts, health information management, and information systems professionals, led by a dually trained surgical resident physician and informatics fellow. Designs and application prototypes were reviewed and revised iteratively on a weekly basis for approximately 7 months until accepted by the project team.

\section{Users}

The primary end users were defined as providers admitting and taking care of patients with adhesive SBOs including but not limited to surgical residents, physician assistants, and surgical attendings. Access to the pathway was not limited, however, to assure that all care providers could also see where the patients were on the pathway. For example, nurses can track their patients on the pathway and actively participate in the patient's care. Pharmacists also have access and can review any medications ordered and administered and the care coordination team can also review the patient's progress on the pathway to aid in discharge planning.

\section{Team}

The development team was comprised of several expert stakeholders: Six clinical content experts (C.M., C.M., J.B., 


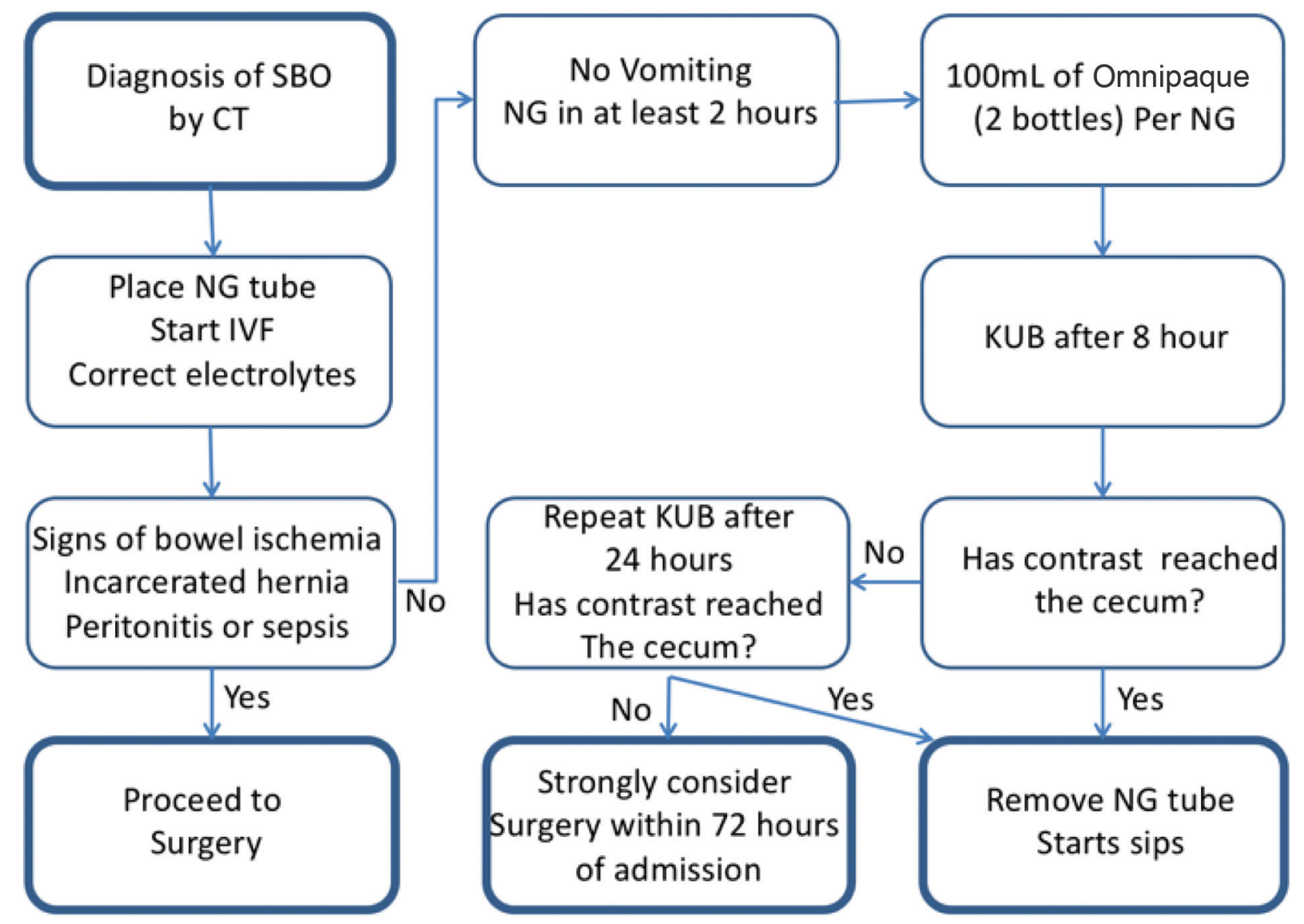

Fig. 1 Paper-based adhesive small bowel obstruction (SBO) pathway.

A.K., H.L., J.H.) and the technical team (A.L., N.P., S.M., A.B., N.D., A.P.). The clinical content lead (H.L.) acted as a bridge for communication between the end users and the development team and also acted as a test user for the application.

\section{Design}

Regular meetings were conducted with the Brigham and Women's Hospital clinical pathways oversight committee which included the clinical context experts as well as hospital leadership to provide feedback on the pathway implementation plan. Weekly meetings were held among the development team to provide feedback throughout the development of the application. Testing was conducted throughout development and at regular intervals after the initial Web application was created. The electronic SBO pathway was constantly tested for functionality and usability.

Throughout the development of the electronic pathway, the core team communicated with the end users through presentations and small focus groups. Several important system features were emphasized by the end users to increase usability and viability: (1) Integration with the EHR: Users wanted a system that was readily accessible through the EHR; (2) Automation of data entry: Residents and physicians desired a completely automated system with seamless data transfer between the application and the
EHR; (3) Real-time data: Users wanted a system that could provide clinical decision support with access to real-time data; and (4) Prospective data collection: Users wanted all pathway compliance data tracked through the application to be exportable to raw data format.

\section{Development}

Based on all of the stakeholders' goals, the decision was made to create an electronic dashboard accessible from the patient's electronic record with autopopulated fields showing progression through the SBO pathway.

The pathways system was implemented as a React.js/.Net Web application with a SQL Server database. The application seamlessly integrates into the EHR's user interface with user and patient context. The application retrieves real-time clinical data via Web services. The clinical and analytical leadership of the project defined a decision support logic for each data point representing the pathway. The system executed this logic to automatically populate data points from the EHR with an option for clinician to review and override. The application monitors user activities and tracks changes in the patient data over time for subsequent data export and analytics.

Three major components were designed and developed:

(1) A dashboard with a list of patients enrolled in the pathway. Patients are automatically enrolled into a pathway 


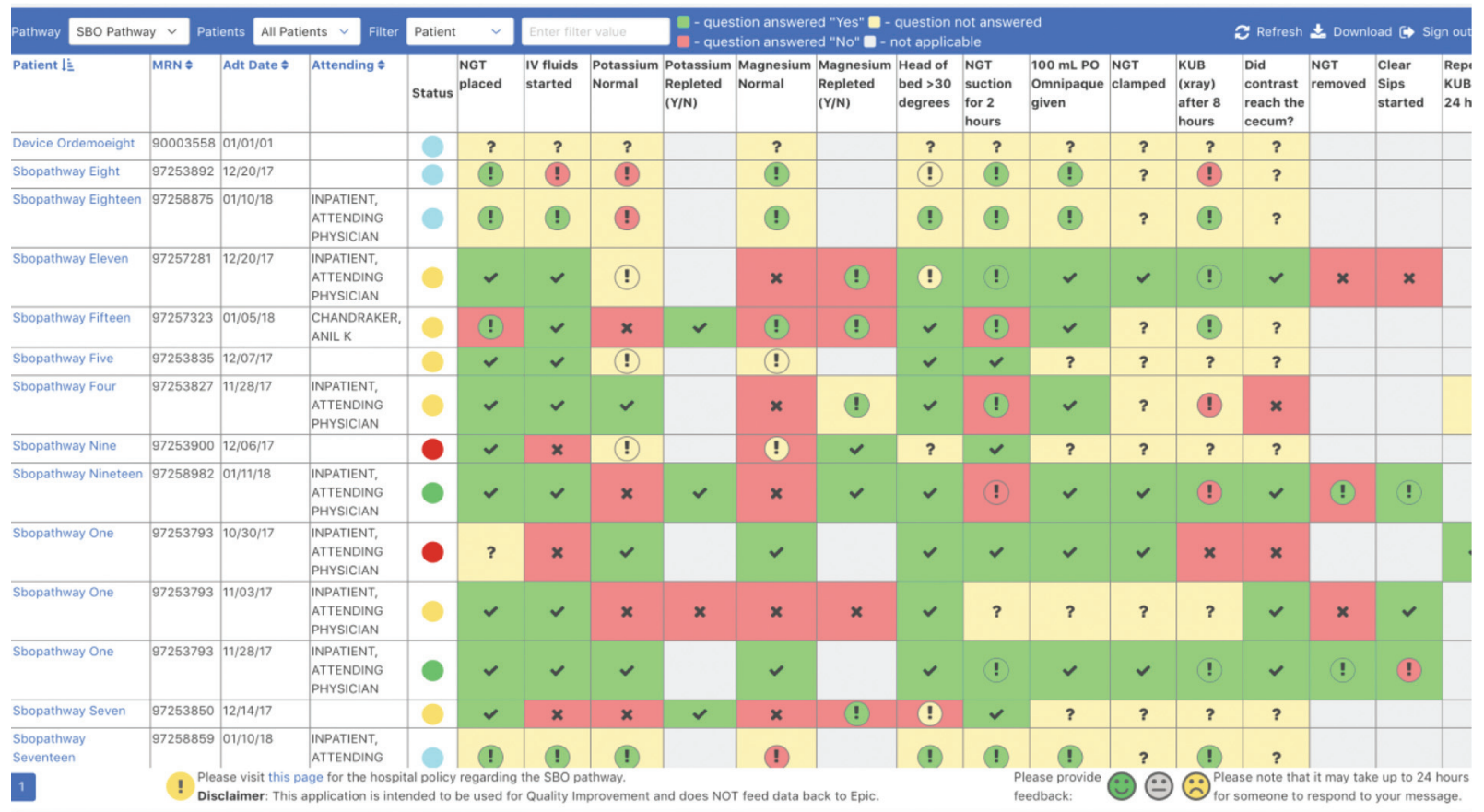

Fig. 2 Small bowel obstruction (SBO) pathway dashboard.

based on the admitting diagnosis of adhesive SBO to the trauma and acute care surgery service with an option for manual enrollment and unenrollment (-Fig. 2).

(2) A form for collecting pathway-specific clinical data points. These data points are exposed on the pathway dashboard and may be autopopulated by the system or manually entered by a clinician. Clinicians have a customized view of the patient data in the EHR directly relevant to the data points being collected. Side bar widgets that provided additional clinical information relevant to the SBO pathway were populated by the EHR data and were also available to the user (-Fig. 3). (3) An administrative component for configuration of a wide range of pathway parameters, including diagnostic codes, list of target data points, and many others. Clinicians and administrators can manage, monitor, and make important changes to the application and individual pathways with minimal involvement of software developers. During the study period, clinicians and administrators did not make any changes to the pathway application.

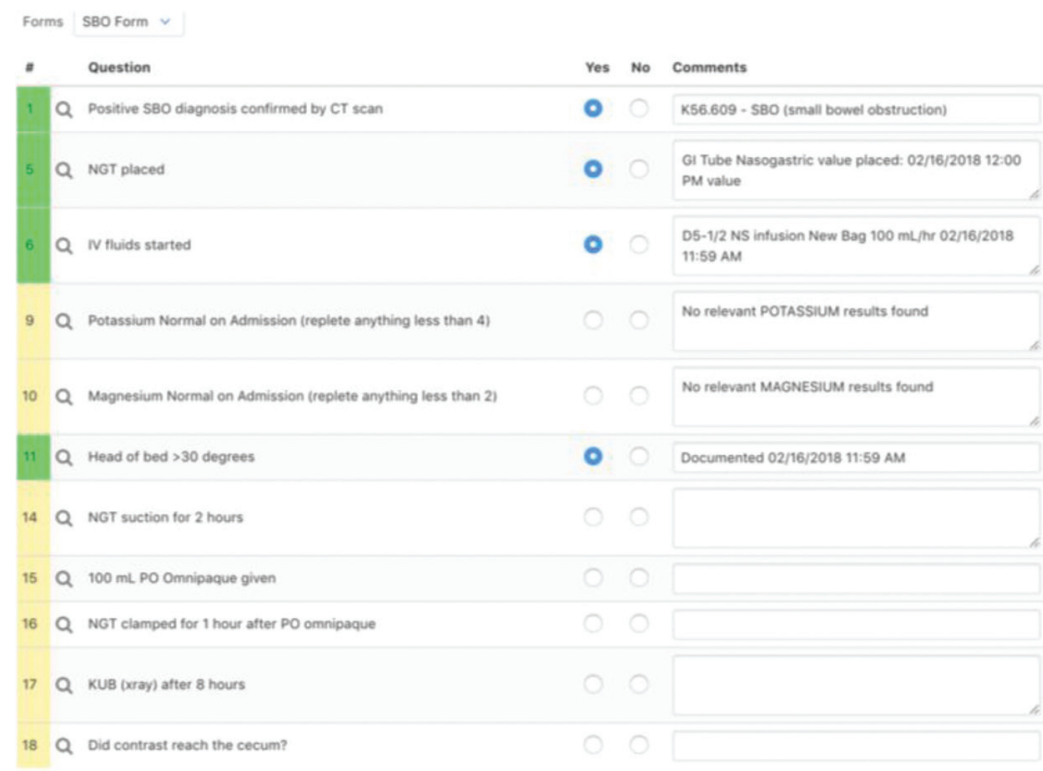

\section{(3) Patient data}
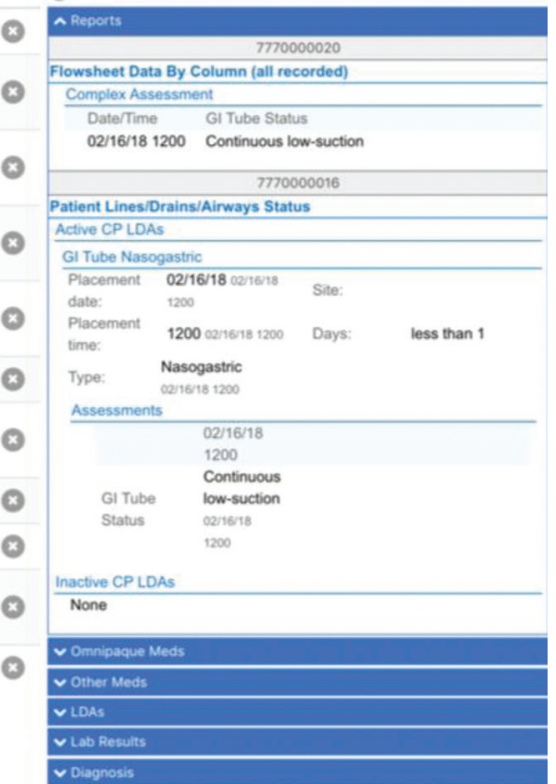

Fig. 3 A form for collecting pathway-specific clinical data points. 


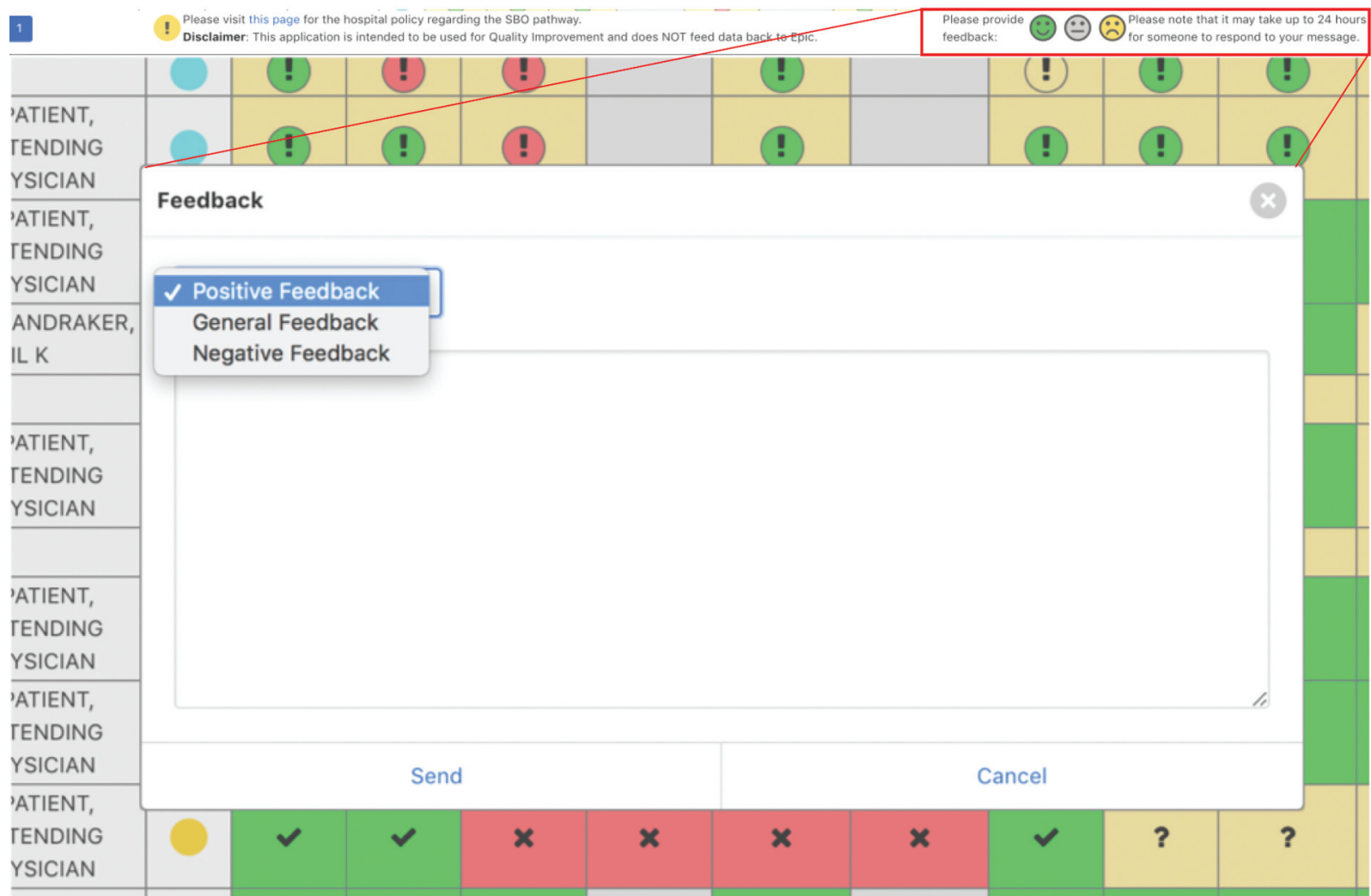

Fig. 4 Feedback button to send messages to the design team.

The electronic pathway also included functionality to allow users to send feedback directly to the development team as well as access data for all SBO pathway patients through a raw data format ( - Fig. 4).

\section{Implementation}

The electronic SBO pathway was implemented in March 2018. The development team continued to meet weekly for approximately 3 months after the implementation to discuss user feedback, software bugs, and improvements.

\section{Evaluation of Electronic SBO Pathway}

All patients admitted to the trauma and acute care surgery service from March 2018 through October 2018 with a diagnosis of adhesive SBO were enrolled in and monitored on the electronic SBO pathway. These patients were prospectively followed and compared with patients on the paper pathway from January 2017 through January 2018 and nonpathway patients from September 2013 through December 2014. Patients on the paper-based SBO pathway prior to January 2017 were excluded from the study to mitigate any noncompliance in the first year of pathway implementation.

Demographic variables including age, gender, body mass index (BMI), and race were collected. Outcomes measured included LOS, readmission, surgery, TTS, and need for bowel resection.

Comparative analyses were completed with Pearson's chisquared test for categorical variables including gender, race, readmission, need for surgery, and bowel resection; analysis of variance test for continuous variables such as age and BMI; and Kruskal-Wallis test for nonparametric continuous variables such as LOS and TTS. Statistical significance was set at a $p$-value of less than 0.05 . This study was approved by the Partners HealthCare Institutional Review Board.

\section{Results}

The electronic SBO pathway is integrated into our EHR, pulling real-time data to prepopulate the evidence-based paper-based algorithm ( Fig. 2). The electronic pathway serves as a clinical decision support tool as well as a reporting tool. It allows providers to prospectively track individual patient progress through the algorithm and guide treatment. It also serves as a tool for providers to review all SBO pathway patient data to monitor compliance. This application simplifies the data transfer process by allowing users to extract the data in a raw data format.

Following initial implementation of the electronic pathway, data for patients who were placed on the electronic SBO pathway application were reviewed. There were 46 patients enrolled in the electronic SBO pathway from March through October 2018. These patients were compared with 93 patients on the paper pathway and 101 nonpathway patients. There were fewer white and more black patients in the paper pathway cohort and this difference was statistically significant. There were no other statistically significant differences in demographics among these groups ( - Table 1 ).

- Table 2 compares the outcomes among the three groups. The median LOS was lower in the electronic and paper pathway cohort who did not have surgery compared with 
Table 1 Patient demographics

\begin{tabular}{|c|c|c|c|c|}
\hline & No pathway $(n=101)$ & Paper pathway $(n=93)$ & Electronic pathway $(n=46)$ & $p$-Value \\
\hline Age (y) [range] & $62[21-101]$ & $64[30-105]$ & $68.5[32-106]$ & 0.0869 \\
\hline \multicolumn{5}{|l|}{ Gender $(n[\%])$} \\
\hline Female & $44[43.6]$ & $43[46.2]$ & 20 [43.5] & 0.919 \\
\hline \multicolumn{5}{|l|}{ Race $(n[\%])$} \\
\hline White & 89 [88.1] & $58[62.4]$ & $37[80.4]$ & $<0.001$ \\
\hline Black & 9 [8.9] & 20 [21.5] & $7[15.2]$ & \\
\hline Other & $3[3.0]$ & $11[11.8]$ & $2[4.3]$ & \\
\hline Unknown & 0 & $4[4.3]$ & 0 & \\
\hline BMI [range] & $25.1[16.6-38.8]$ & $25.8[14.7-53.1]$ & $26.0[17.5-41.3]$ & 0.467 \\
\hline
\end{tabular}

Abbreviation: BMI, body mass index.

Table 2 Comparative analysis of outcomes

\begin{tabular}{|l|l|l|l|l|}
\hline & No pathway & Paper pathway & Electronic pathway & $p$-Value \\
\hline Length of stay (LOS), median days [range] & $4[1-35]$ & $3[1-32]$ & $3.5[1-19]$ & 0.09 \\
\hline Surgery & $12[2-35]$ & $13[6-32]$ & $8[4-19]$ & 0.158 \\
\hline No surgery & $4[1-13]$ & $3[1-27]$ & $3[1-11]$ & 0.04 \\
\hline Time to surgery, median days [range] & $3[0-15]$ & $3[0-10]$ & $2[1-5]$ & 0.822 \\
\hline Surgery needed, $n[\%]$ & $19[18.8]$ & $12[12.9]$ & $10[21.7]$ & 0.356 \\
\hline $\begin{array}{l}\text { Bowel } \\
\text { Resection, } n[\%]\end{array}$ & $6[31.6]$ & $4[33.3]$ & $4[40]$ & 0.900 \\
\hline Readmission, $n[\%]$ & $7[6.9]$ & $8[8.6]$ & $1[2.2]$ & 0.356 \\
\hline
\end{tabular}

those not on either pathway ( 3 days [range 1-11] vs. 3 days [range 1-27] vs. 4 days [range 1-13], $p=0.04$ ). Median LOS was lower in the electronic pathway cohort compared with the paper pathway and nonpathway cohorts who underwent surgery but this finding did not reach statistical significance (8 days [range 4-19] vs. 13 days [range 6-32] vs. 12 days [range 2-35], $p=0.158$ ). TTS, rates of readmission, surgery, and bowel resection were not significantly different among the three cohorts.

\section{Discussion}

Previous studies have shown that an SBO treatment pathway using low-osmolar contrast, Omnipaque, is associated with a decreased hospital LOS for patients with adhesive SBOs. ${ }^{15,17}$ This study is an example of how an electronic clinical pathway can improve treatment efficiency and patient outcomes. $^{2}$ Following the successful implementation of the paper-based SBO pathway, an electronic dashboard was built to better visualize and audit patients enrolled in the pathway. In this study, we showed that it is feasible to implement and utilize an electronic clinical pathway for evidence-based, widely utilized pathways with similar outcomes to the paper pathway.

Our findings support previous literature that demonstrates the usability of electronic clinical pathways. Many studies previously described the utility of visual and interac- tive clinical pathway platforms in providing real-time data on patients, aiding prospective data collection for researchers, engaging patients with meaningful data, and further optimizing future care patterns. ${ }^{18,19}$ The electronic SBO pathway performed all of these functions real time and provided multiple stakeholders with useful and powerful data to support clinical decision-making and better integrate evidence-based guidelines in to the institutional practice. ${ }^{20}$ Studies have shown that successful integration and implementation of clinical decision support tools such as these can improve clinical processes and outcomes and reduce errors. ${ }^{21-23}$

The development of the electronic pathway required significant involvement of various stakeholders. The multidisciplinary team included clinicians, quality and safety experts, health information management, and information systems professionals. After achieving a consensus on goals for the project, multiple designs of the user interface and application prototypes were reviewed on a weekly basis until a final, working product was accepted by all members of the team. We followed previously cited best practices on the development and implementation of clinical pathways that showed that implementation of an evidence-based clinical pathway requires: (1) clinician involvement, (2) evidencebased intervention, (3) local consensus, (4) multidisciplinary implementation team, (5) identification of potential barriers to change, and (6) educational outreach. ${ }^{22}$ 
Following the implementation, the pathway went through several iterative updates to address any software bugs as well as feedback from the users. Evaluation of the patients enrolled in the electronic pathway over an 8-month period showed no negative impact on clinical outcomes. This demonstrates safety and feasibility of implementing an electronic pathway. Nevertheless, we were unable to demonstrate a clear benefit of implementing an electronic pathway compared with a paperbased pathway. This demonstrated lack of clear benefit may be a barrier for those who have yet to implement electronic versions of evidence-based paper pathways and need justification to generate funding and resources for the development and implementation.

One reason for this lack of difference in outcomes between the paper and electronic pathways may be that the use of the electronic pathway required several additional clicks outside of the users' normal workflow to open and view the dashboard. While these actions required very little time, it was difficult for clinicians to adapt their workflow enough to have meaningful integration of the pathway as a clinical decision support tool. This is supported by previous studies that have shown that extra workload may hinder adoption of computerized decision support systems. ${ }^{24}$ Given these limitations, our pathway seemed to be more useful as a reporting and prospective data collection tool rather than a real-time clinical decision support tool which may have led to a lack of difference in outcomes. Continued education on the importance of the clinical pathway may lead to increased adoption in the future. Also, further developments to fully integrate the electronic SBO pathway in to the EHR and the clinician workflow may lead to wider adoption and acceptance. Nevertheless, this level of integration still requires the end user to remember to launch the application, know where to launch it, and to successfully execute these steps. We have shown this may be a difficult workflow for busy clinicians caring for a large service. Better overall integration of thirdparty Web applications into the clinician's EHR workflows is needed. The CDS Hooks initiatives is a good solution that will enable adding clinical content into decision support. ${ }^{25}$ While federal policy is working to make more data available via APIs, we also need more standardized methods of integrating Web applications into EHRs. In the short term, we are now exploring whether we can leverage our improved out-of-thebox vendor features to deliver an electronic SBO pathway.

\section{Limitations}

There are several limitations to this study. This is a single institution review of a combination of retrospective sample and prospectively collected data. The use of retrospective data can introduce unintended bias to the analysis. There were also long time periods between the three comparison groups that may benefit from tests for trend in proportions to better account of changes that occurred outside the context of this study. Also, the sample of patients on the electronic pathway was much smaller given the shorter duration of time for which data were available; increasing the sample size may power findings that were not able to reach statistical significance. Case-matching is a methodology that could have been used to address bias in our study; however, our ability to match was limited by the small sample sizes of our cohorts. We developed a custom electronic pathway system which limits the generalizability of our results. Additionally, while our API approach is applicable to other applications being built on top of EHRs, we did not use standards-based APIs (FHIR APIs). While we incorporated user feedback through informal inquiry, we did not do any formal usability testing. Future implementations of other SBO pathways will include formal usability testing with end users. Finally, widespread adoption of new technology or pathway is often met with resistance and affected by low compliance rates when initially studied. Future studies using data for the electronic pathway after increased adoption are warranted.

\section{Conclusion}

Our findings demonstrate the feasibility of developing and implementing an electronic version of an evidence-based clinical pathway for adhesive SBOs without impacting outcomes. Despite no significant improvement in clinical outcomes from the previous paper-based pathway, the electronic pathway has been integral in data collection and prospective tracking of SBO pathway metrics. Future iterations to further integrate the SBO and other pathways in to the end-users' workflow may demonstrate increased improvements in clinically meaningful outcomes.

\section{Clinical Relevance Statement}

It is feasible to develop evidence-based electronic pathways that adhere to national guidelines to standardize care for surgical patients. These electronic apps can be integral in prospective data collection and feedback to improve clinical outcomes. Institutions should adopt development of electronic apps for existing clinical pathways that have already proven to be successful.

\section{Multiple Choice Questions}

1. A patient presents to the emergency department with abdominal pain, nausea, and vomiting. Imaging shows evidence of an adhesive small bowel obstruction. Based on recent literature, the patient should be placed on a clinical pathway utilizing water-soluble contrast. What is the major benefit of a clinical pathway?

a. Clinical pathways are widely utilized and have been associated with decreased complications, length of stay, and overall costs of care.

b. Clinical pathways reduce the need for multidisciplinary teams.

c. Clinical pathways increase provider and patient interaction.

d. Clinical pathways are not utilized by many institutions and can be a novel approach to medical care. 
Correct Answer: The correct answer is option a. In Garbin's landmark article, he introduces clinical pathways as a comprehensive method of planning, delivering, and monitoring patient care to improve quality outcomes while decreasing cost and increasing efficiency. Since then, clinical pathways in some form have been implemented in more than $80 \%$ of hospitals in the United States. They have been proven to be safe and there is a great opportunity to increase the utility of clinical pathways with the integration of health information technology.

2. Current guidelines recommend limiting nonoperative therapy for adhesive small bowel obstructions to 3 to 5 days. Recent literature has demonstrated the benefits of administering water-soluble contrast through a nasogastric tube. This pathway is implemented in a high-volume academic center in which there are over 100 adhesive small bowel obstructions admitted annually but it has yet to be integrated in to the electronic health record system. Which of the following is the appropriate next step after the successful initial implementation of the small bowel obstruction pathway?

a. Continue using the paper pathway as it has been successful and there is no need for change.

b. Implement an electronic pathway to better evaluate the process metrics and outcome measures.

c. Stop using the pathway altogether.

d. Perform a randomized control trial between the paper pathway and no pathway.

Correct Answer: The correct answer is option b. The implementation of an electronic pathway is very important to help clinicians make decisions about patients in real time. Also, electronic pathways can be used to collect real-time patient data that can be used to drive the development of models and tools to audit and revise current workflows and improve evidence-based care delivery.

Protection of Human and Animal Subjects

No human subjects were involved in the project.

Funding

H.L. is supported by the National Library of Medicine Institutional training grant for research training in biomedical informatics and data science (T15) under award number T15LM007092. There were no other funding sources. Corresponding author had full access to all the data in the study and had final responsibility for the decision to submit manuscript.

Conflict of Interest

None declared.

\section{References}

1 Kinsman L, Rotter T, James E, Snow P, Willis J. What is a clinical pathway? Development of a definition to inform the debate. BMC Med 2010;8:31
2 Zhang Y, Padman R. Data-driven clinical and cost pathways for chronic care delivery. Am J Manag Care 2016;22(12):816-820

3 Spiegel TF, Wassermann TB, Neumann N, et al. A clinical pathway for heart failure reduces admissions from the ED without increasing congestion in the ED. Am J Emerg Med 2018;36(07): 1202-1208

4 Than MP, Pickering JW, Dryden JM, et al; ICare-ACS Implementation Group. ICare-ACS (Improving Care Processes for Patients With Suspected Acute Coronary Syndrome): a study of crosssystem implementation of a national clinical pathway. Circulation 2018;137(04):354-363

5 Austrian JS, Volpicelli F, Jones S, et al. The financial and clinical impact of an electronic health record integrated pathway in elective colon surgery. Appl Clin Inform 2020;11(01):95-103

6 Rao G, Kirley K, Epner P, et al. Identifying, analyzing, and visualizing diagnostic paths for patients with nonspecific abdominal pain. Appl Clin Inform 2018;9(04):905-913

7 Catena F, Di Saverio S, Coccolini F, et al. Adhesive small bowel adhesions obstruction: evolutions in diagnosis, management and prevention. World J Gastrointest Surg 2016;8(03):222-231

8 Azagury D, Liu RC, Morgan A, Spain DA. Small bowel obstruction: a practical step-by-step evidence-based approach to evaluation, decision making, and management. J Trauma Acute Care Surg 2015;79(04):661-668

9 Keenan JE, Turley RS, McCoy CC, Migaly J, Shapiro ML, Scarborough JE. Trials of nonoperative management exceeding 3 days are associated with increased morbidity in patients undergoing surgery for uncomplicated adhesive small bowel obstruction. J Trauma Acute Care Surg 2014;76(06):1367-1372

10 Ceresoli M, Coccolini F, Montori G, Sartelli M, Catena F, Ansaloni L. Water-soluble contrast agent in adhesive small bowel obstruction: the game is still on. Surgery 2017;162(01):199-200

11 Ceresoli M, Coccolini F, Catena F, et al. Water-soluble contrast agent in adhesive small bowel obstruction: a systematic review and meta-analysis of diagnostic and therapeutic value. Am J Surg 2016;211(06):1114-1125

12 Assalia A, Schein M, Kopelman D, Hirshberg A, Hashmonai M. Therapeutic effect of oral Gastrografin in adhesive, partial smallbowel obstruction: a prospective randomized trial. Surgery 1994; 115(04):433-437

13 Abbas S, Bissett IP, Parry BR. Oral water soluble contrast for the management of adhesive small bowel obstruction. Cochrane Database Syst Rev 2007;(03):CD004651

14 Zhang Y, Padman R. An interactive platform to visualize datadriven clinical pathways for the management of multiple chronic conditions. Stud Health Technol Inform 2017;245:672-676

15 Lyu HG, Castillo-Angeles M, Bruno M, et al. Outcomes of a lowosmolar water-soluble contrast pathway in small bowel obstruction. J Trauma Acute Care Surg 2019;87(03):630-635

16 Mandl KD, Kohane ISA. A 21st-century health IT system - creating a real-world information economy. N Engl J Med 2017;376(20): 1905-1907

17 Trevino CM, VandeWater T, Webb TP. Implementation of an adhesive small bowel obstruction protocol using low-osmolar water soluble contrast and the impact on patient outcomes. Am J Surg 2019;217(04):689-693

18 Rodriguez-Loya S, Aziz A, Chatwin C. A service oriented approach for guidelines-based clinical decision support using BPMN. Stud Health Technol Inform 2014;205:43-47

19 Zhang Y, Padman R, Patel N. Paving the CoWpath: learning and visualizing clinical pathways from electronic health record data. J Biomed Inform 2015;58:186-197

20 Sahota N, Lloyd R, Ramakrishna A, et al; CCDSS Systematic Review Team. Computerized clinical decision support systems for acute care management: a decision-maker-researcher partnership systematic review of effects on process of care and patient outcomes. Implement Sci 2011;6(01):91-98 
21 Griffey RT, Lo HG, Burdick E, Keohane C, Bates DW. Guided medication dosing for elderly emergency patients using realtime, computerized decision support. J Am Med Inform Assoc 2012;19(01):86-93

22 Hemens BJ, Holbrook A, Tonkin M, et al; CCDSS Systematic Review Team. Computerized clinical decision support systems for drug prescribing and management: a decision-maker-researcher partnership systematic review. Implement Sci 2011;6(01):89

23 Rotter T, Plishka C, Hansia MR, et al. The development, implementation and evaluation of clinical pathways for chronic ob- structive pulmonary disease (COPD) in Saskatchewan: protocol for an interrupted times series evaluation. BMC Health Serv Res 2017;17(01):782

24 Horvath KJ, Ecklund AM, Hunt SL, Nelson TF, Toomey TL. Developing Internet-based health interventions: a guide for public health researchers and practitioners. J Med Internet Res 2015; 17(01):e28

25 CDS Hooks [Internet], Ann Arbor \& Boston: HL7 \& Boston Children's Hospital; 2019. Available at: https://cds-hooks.hl7.org/. Accessed March 2, 2020 